\title{
Maternal plasma DNA testing for aneuploidy in pregnancies achieved by assisted reproductive technologies
}

\author{
Geralyn Lambert-Messerlian, PhD¹, Edward M. Kloza, MS1', John Williams III, MD², Jaroslav Loucky, PhD³, \\ Barbara O'Brien, MD, Louise Wilkins-Haug, MD, PhD ${ }^{5}$, Maurice J. Mahoney, $\mathrm{MD}^{6}$, \\ Pierangela De Biasio, $\mathrm{MD}^{7}$, Antoni Borrell, $\mathrm{MD}^{8}$, Mathias Ehrich, MD9, Dirk van den Boom, PhD ${ }^{9}$, \\ Allan T. Bombard, MD ${ }^{9,10}$, Cosmin Deciu, $\mathrm{MS}^{10}$ and Glenn E. Palomaki, PhD ${ }^{1}$
}

Purpose: We sought to compare measurements of circulating cellfree DNA as well as Down syndrome test results in women with naturally conceived pregnancies with those conceived using assisted reproductive technologies.

Methods: Data regarding assisted reproductive technologies were readily available from seven enrollment sites participating in an external clinical validation trial of nested case/control design. Measurements of circulating cell-free fetal and total DNA, fetal fraction (ratio of fetal to total DNA), chromosome-specific $z$-scores, and karyotype results were available for analysis.

Results: Analyses were restricted to 632 euploid (5.2\% assisted reproductive technologies) and 73 Down syndrome (13.7\% assisted reproductive technologies), including 16 twin pregnancies. No differences were found for fetal or total circulating cell-free DNA, or for the fetal fraction in euploid $(P=0.70)$ or Down syndrome $(P=0.58)$ pregnancies by method of conception. There appeared to be systematic $z$-score reductions for chromosomes 21,18 , and 13 in assisted reproductive technologies versus natural euploid pregnancies $(P=$ $0.048,0.0032$, and 0.36 , respectively).

Conclusion: Assisted reproductive technologies and naturally conceived pregnancies contribute similar levels of circulating cell-free DNA into maternal circulation. Small differences in the $z$-scores of pregnancies achieved by assisted reproductive technologies were observed and do not appear to be test-related artifacts. However, the findings need confirmation before any consideration of changes to testing and reporting protocols.

Genet Med advance online publication 3 October 2013

Key Words: aneuploidy screening; assisted reproductive technologies; circulating cell-free DNA; Down syndrome; fetal fraction
Advances in molecular technologies have resulted in the introduction of next-generation sequencing of maternal plasma DNA for prenatal identification of Down syndrome and other common trisomies. ${ }^{1,2}$ Circulating cell-free (ccf) DNA of placental/fetal origin ${ }^{3}$ in the maternal circulation is thought to be released by apoptotic cells of the placenta, ${ }^{4}$ and the success of next-generation sequencing is dependent on the percentage of fetal (placental) DNA present in maternal plasma (the fetal fraction). The higher the fetal fraction, the more easily a trisomy or other aneuploidy can be detected. On average, 13\% of ccf DNA in maternal plasma is of fetal (placental) origin, and fetal fractions of less than $4 \%$ may not be sufficient for reliable testing. ${ }^{1}$

First and second trimester maternal serum marker levels are known to be altered in pregnancies conceived using assisted reproductive technologies (ART) versus those conceived naturally (controls). ${ }^{5-10}$ In particular, secretory products of the placenta, such as human chorionic gonadotropin and inhibin A, are $10-30 \%$ higher in pregnancies from ART relative to control pregnancies. The cause of the increased secretion is not known. Regardless, serum marker levels can be adjusted so that screening performance in ART pregnancies is comparable with that of controls. Given that a portion of ccf DNA also is released by placental cells, albeit by a different mechanism, we sought to explore the possibility that the levels of ccf DNA also differed between $\mathrm{ART}$ and control pregnancies. If ART pregnancies were to release at least as much fetal-specific ccf DNA into maternal blood as do naturally conceived pregnancies, this could reassure clinicians that next-generation testing in ART pregnancies would perform at least as well as in naturally conceived pregnancies. In addition, we examined the chromosome-specific $z$-score and the final interpretation of the testing in the ART and control pregnancies.

\section{MATERIALS AND METHODS}

Samples for this study were selected from among those reported earlier as part of a multicenter international clinical validation of a next-generation sequencing test for Down syndrome. ${ }^{1}$

${ }^{1}$ Department of Pathology and Laboratory Medicine, Women and Infants Hospital, Alpert Medical School of Brown University, Providence, Rhode Island, USA; ${ }^{2}$ Department of Obstetrics and Gynecology, Cedars-Sinai Medical Center, Los Angeles, California, USA; ${ }^{3}$ IMALAB s.r.o. Medical Laboratories, Zlin, Czech Republic; ${ }^{4}$ Department of Obstetrics and Gynecology, Prenatal Diagnosis Center, Women and Infants Hospital and the Alpert Medical School of Brown University, Providence, Rhode Island, USA; ${ }^{5}$ Department of Obstetrics and Gynecology, Brigham and Women's Hospital, Boston, Massachusetts, USA; ${ }^{6}$ Department of Genetics, Yale University, New Haven, Connecticut, USA; ${ }^{7}$ Istituto G. Gaslini, Genova, Italy; ${ }^{8}$ Hospital Clinic Barcelona, Barcelona, Spain; ${ }^{9}$ Sequenom, San Diego, California, USA; ${ }^{10}$ Sequenom Center for Molecular Medicine, San Diego, California,

USA. Correspondence: Geralyn Lambert-Messerlian (gmesserlian@wihri.org) 
Before an invasive diagnostic procedure, plasma was collected for quantification of the fetal fraction and subsequent DNA sequencing. ${ }^{1,3}$ The original study design was a nested case/ control with each Down syndrome case being matched with seven euploid control samples. A retrospective chart review was performed at 7 of the 27 enrollment sites participating in the original study which had active IRB approval and data recorded on the use of ART (Women and Infants Hospital, Providence, RI; Cedars-Sinai Prenatal Diagnostic Center, Los Angeles, CA; IMALAB s.r.o. Medical Laboratories and Prediko s.r.o. Genetic and Prenatal Screening Centre, Zlin, Czech Republic; Brigham and Women's Hospital, Boston, MA; Yale University, New Haven, CT; Istituto G. Gaslini, Genova, Italy; and Hospital Clinic Barcelona, Barcelona, Catalonia, Spain). Information on the use of ART was not available for all enrolled women from each site.

Results were compared by Welch's $t$-test due to the potential for unequal variances and the large differences in the number versus naturally conceived pregnancies. ${ }^{11}$ Gestational age, maternal weight, and maternal age were assumed to have a reasonably normal distribution. This was also true for the $z$-scores in euploid singleton (and twin) pregnancies, which are expected to be normally distributed with a mean of 0 and standard deviation of $1 .{ }^{1,2,11,12}$ Fetal fraction results, and $z$-scores in Down syndrome pregnancies, were subjected to a logarithm transformation before comparison, as these data are right skewed. ${ }^{13}$ Proportions were compared using Fisher's exact tests. Significance was two tailed at $P<0.05$; no adjustment for multiple comparisons was made.

\section{RESULTS}

A total of 740 patients who had DNA sequencing also had information available about whether ART was used. Among these, 44 pregnancies (5.9\%; 95\% CI: 4.3-7.9\%) were conceived by ART.
The rate of ART pregnancies at the seven centers ranged from 2.4 to $15 \%$, with higher observed rates among the US centers compared with the international centers ( 8.4 and $5.1 \%$, respectively, $P=0.071)$. Among the 740 pregnancies, 73 were associated with Down syndrome, 18 with trisomy 18, 3 with trisomy 13 , and 14 with other aneuploidies. All but the Down syndrome cases were excluded from the formal analyses due to very few observations for reliable conclusions. This left 43 ART and 662 naturally conceived pregnancies for study. Among these, the vast majority $(93 \%$; 41 of 43$)$ of ART pregnancies used in vitro fertilization, only one of which included an egg donation. Two additional pregnancies reported use of intrauterine insemination. No information was available regarding the number of embryos transferred. Multiple gestation pregnancies have been reported to have higher fetal fractions. ${ }^{12,14}$ Therefore, group comparisons of ccf DNA levels and fetal fractions were conducted after excluding data from the 16 sets of twins. The twin results were, however, included in the analyses of demographic information and $z$-scores.

Table 1 shows demographic and DNA-related measurements in the ART and naturally conceived pregnancies, after stratification into euploid and Down syndrome outcomes. Maternal age, gestational age, and maternal weight were not different between all ART and naturally conceived pregnancies tested in either outcome group. Similar levels of total DNA and fetal DNA were also found between ART and naturally conceived singleton euploid pregnancies, as well as in the Down syndrome pregnancies. The geometric mean fetal fractions of 14.0 and $13.5 \%$ in ART and naturally conceived euploid pregnancies were not significantly different $(P=0.70)$. The fetal fractions of 13.0 and $14.9 \%$ for those same group of Down syndrome pregnancies were also not significantly different $(P=0.58)$. Both of these fetal fractions were somewhat lower than the $15.2 \%$ reported in all 212 singleton pregnancies with Down syndrome from the

Table 1 Maternal demographics and circulating cell-free DNA levels in euploid and Down syndrome pregnancies, stratified by whether or not conception was achieved using ART

\begin{tabular}{|c|c|c|c|c|c|c|}
\hline \multirow[b]{3}{*}{ Characteristic } & \multicolumn{3}{|c|}{ Euploid pregnancies $^{\mathrm{a}}$} & \multicolumn{3}{|c|}{ Down syndrome pregnancies ${ }^{b}$} \\
\hline & ART $(N=33)$ & Natural $(N=599)$ & \multirow[b]{2}{*}{$P$} & ART $(N=10)$ & Natural $(N=63)$ & \multirow[b]{2}{*}{$P$} \\
\hline & \multicolumn{2}{|c|}{ Mean value (range)c } & & \multicolumn{2}{|c|}{ Mean value (range) } & \\
\hline Maternal weight (lbs) & $144(99-225)$ & $146(97-321)$ & 0.69 & $149(125-183)$ & $146(101-233)$ & 0.72 \\
\hline Maternal age (years) & $37.8(29-45)$ & $36.9(19-46)$ & 0.29 & $38.4(32-42)$ & $36.8(23-46)$ & 0.34 \\
\hline Fetal DNA (copies/ml)d & $116(71-233)$ & $116(25-1764)$ & 0.96 & $157(63-368)$ & $132(28-593)$ & 0.50 \\
\hline Fetal fraction (\%) ${ }^{d}$ & $14.0(4-30)$ & $13.5(4-47)$ & 0.70 & $13.0(5-24)$ & $14.9(4-38)$ & 0.58 \\
\hline z-Score & \multicolumn{2}{|c|}{ Arithmetic mean (range) } & \multicolumn{4}{|c|}{ Geometric mean (range) } \\
\hline Chromosome 21 & $-0.38(-1.86-0.67)$ & $-0.13(-3.89-2.12)$ & 0.048 & $8.7(4.49-19.3)$ & $11.4(2.27-28.3)$ & 0.17 \\
\hline Chromosome 18 & $-0.45(-1.73-0.87)$ & $-0.02(-4.11-3.46)$ & 0.0032 & $0.60(-1.03-0.96)$ & $0.50(-5.14-1.59)$ & 0.63 \\
\hline
\end{tabular}

ART, assisted reproductive techniques.

ancludes five ART and five natural twin euploid pregnancies. 'Includes three ART and three natural twin Down syndrome pregnancies. 'For the first three variables, the

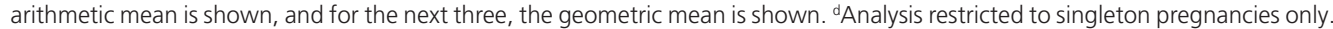


primary study, ${ }^{1}$ regardless of ART status. These findings were not changed by exclusion of the two ART pregnancies achieved by intrauterine insemination.

Among the euploid pregnancies, no false-positive Down syndrome results occurred. Among the 73 Down syndrome pregnancies, there was one false negative ( $z$-score: 2.27$)$, and it occurred among the naturally conceived pregnancies. There was only one false-positive chromosome 18 ( $z$-score: 3.46$)$ and it occurred among the 599 naturally conceived euploid pregnancies. There were two false-positive chromosome 13 results in euploid pregnancies: one among the ART pregnancies and one among the naturally conceived pregnancies.

Table 1 also shows summary $z$-score information for chromosomes 21, 18, and 13. As expected, the $z$-scores in euploid naturally conceived pregnancies were centered close to 0 for all three chromosomes. However, the $z$-scores for chromosomes 21,18 , and 13 were all lower in euploid ART pregnancies, with the reductions for chromosomes 21 and 18 reaching statistical significance ( $P=0.048$ and 0.0032 , respectively). Among Down syndrome pregnancies, the chromosome $21 z$-scores were also lower on average in ART compared with natural pregnancies (geometric means 8.7 and 11.4, respectively), but this did not reach statistical significance $(P=0.17)$. As a separate observation, the $z$-scores in Down syndrome cases for chromosome 18 and 13 tended to be higher than the expected $z$-score of 0 . This is consistent with our earlier findings that some Down syndrome cases were associated with slightly positive results for other chromosomes (double positives). ${ }^{1}$

A subset of these samples were part of an internal validation study of an updated laboratory-developed test ${ }^{15}$ that included automated library preparation, higher sample multiplexing during sequencing, and improved bioinformatic tools. Among euploid samples, the point estimate for natural versus in vitro fertilization average $z$-scores for the three chromosomes were reduced, but none reached statistical significance $(P=0.077$, 0.48 , and 0.25 , for chromosomes 21,18 , and 13 , respectively).

A total of 18 trisomy 18 test results were included in our dataset and of these, only one was conceived via ART and none were multiple gestations. The geometric mean fetal fractions for the 17 naturally conceived and one ART trisomy 18 were 7.8 and $3.6 \%$, respectively. The chromosome $18 z$-scores were 8.9 and 6.4, respectively. Among the 3 trisomy 13 samples, none were conceived via ART and the geometric mean fetal fraction and trisomy $13 z$-score in the naturally conceived pregnancies was 11.1 and $19.9 \%$, respectively.

\section{DISCUSSION}

The present results do not show increased circulating cellfree fetal DNA in pregnancies achieved using ART, either in absolute levels or by the fetal fraction. This is in agreement with another study that found no increase in the amount of cell-free fetal DNA in maternal plasma from pregnancies conceived by in vitro fertilization relative to controls. ${ }^{16}$ On the basis of detection and false-positive rates, next-generation sequencing of maternal plasma for Down syndrome appears to perform similarly in ART and naturally conceived pregnancies. This has also been reported in a limited study of 38 ART pregnancies, ${ }^{2}$ that detected all 17 aneuploidies with no false-positive results. However, examining the detection and false-positive rates alone is an insensitive analytic method for identifying systematic differences. Instead, we compared the distribution of $z$-scores in ART and naturally conceived pregnancies and identified small but statistically significant reductions among euploid ART pregnancies. A reduction also occurred for the Down syndrome ART pregnancy group, but the difference did not reach statistical significance. The underlying biology as to why this might occur is unclear. It is well known that changes in some serum markers for Down syndrome are different in in vitro fertilization versus naturally conceived pregnancy, and these changes persist into the second trimester. It is also possible that ART pregnancies are more likely to include a vanishing twin and residual placental tissue. If these findings are confirmed, it may be useful to make minor adjustments to the $z$-scores or their interpretation in pregnancies conceived via ART.

\section{ACKNOWLEDGMENTS}

We thank the late Jacob A. Canick, PhD, Director of the Division of Medical Screening and Special Testing, for his assistance in all aspects of preparing this report.

\section{DISCLOSURE}

G.E.P. was a member of the Sequenom Clinical Advisory Board from late 2007 through October 2008. From November 2008 through February 2012, he was co-principal investigator of the InFANet Study (clinicaltrials.gov—NCT00877292) funded through a research agreement between Women \& Infants Hospital and Sequenom. G.L.-M. and E.M.K. also participated in that study. J.W., J.L., B.O.'B., L.W.-H., M.J.M., P.D.B., and A.B. were each site principal investigator for that same study. M.E., D.v.d.B., A.T.B., and C.D. are employees of Sequenom.

\section{REFERENCES}

1. Palomaki GE, Kloza EM, Lambert-Messerlian GM, et al. DNA sequencing of maternal plasma to detect Down syndrome: an international clinical validation study. Genet Med 2011;13:913-920.

2. Bianchi DW, Platt LD, Goldberg JD, Abuhamad AZ, Sehnert AJ, Rava RP MatErnal BLood IS Source to Accurately diagnose fetal aneuploidy (MELISSA) Study Group. Genome-wide fetal aneuploidy detection by maternal plasma DNA sequencing. Obstet Gynecol 2012;119:890-901.

3. Nygren AO, Dean J, Jensen TJ, et al. Quantification of fetal DNA by use of methylation-based DNA discrimination. Clin Chem 2010;56:1627-1635.

4. Alberry M, Maddocks D, Jones $M$, et al. Free fetal DNA in maternal plasma in anembryonic pregnancies: confirmation that the origin is the trophoblast. Prenat Diagn 2007;27:415-418.

5. Wald NJ, White N, Morris JK, Huttly WJ, Canick JA. Serum markers for Down's syndrome in women who have had in vitro fertilisation: implications for antenatal screening. Br J Obstet Gynaecol 1999;106:1304-1306.

6. Lambert-Messerlian G, Dugoff L, Vidaver J, et al. First- and second-trimester Down syndrome screening markers in pregnancies achieved through assisted reproductive technologies (ART): a FASTER trial study. Prenat Diagn 2006;26:672-678.

7. Barkai G, Goldman B, Ries L, Chaki R, Dor J, Cuckle H. Down's syndrome screening marker levels following assisted reproduction. Prenat Diagn 1996;16:1111-1114. 
8. Perheentupa A, Ruokonen A, Tuomivaara L, Ryynänen M, Martikainen H Maternal serum beta-HCG and alpha-fetoprotein concentrations in singleton pregnancies following assisted reproduction. Hum Reprod 2002;17:794-797.

9. Bar-Hava I, Yitzhak M, Krissi H, et al. Triple-test screening in in vitro fertilization pregnancies. J Assist Reprod Genet 2001;18:226-229.

10. Hui PW, Tang MH, Lam YH, Ng EH, Yeung WS, Ho PC. Maternal serum hCG and alpha-fetoprotein levels in pregnancies conceived after IVF or ICSI with fresh and frozen-thawed embryos. Hum Reprod 2003;18:572-575.

11. Sehnert AJ, Rhees B, Comstock D, et al. Optimal detection of fetal chromosomal abnormalities by massively parallel DNA sequencing of cell-free fetal DNA from maternal blood. Clin Chem 2011;57:1042-1049.

12. Canick JA, Kloza EM, Lambert-Messerlian GM, et al. DNA sequencing of maternal plasma to identify Down syndrome and other trisomies in multiple gestations. Prenat Diagn 2012;32:730-734.
13. Canick JA, Palomaki GE, Kloza EM, Lambert-Messerlian GM, Haddow JE. The impact of maternal plasma DNA fetal fraction on next generation sequencing tests for common fetal aneuploidies. Prenat Diagn 2013;33:667-674.

14. Attilakos G, Maddocks DG, Davies T, et al. Quantification of free fetal DNA in multiple pregnancies and relationship with chorionicity. Prenat Diagn 2011;31:967-972.

15. Jensen TJ, Zwiefelhofer T, Tim RC, et al. High-throughput massively parallel sequencing for fetal aneuploidy detection from maternal plasma. PLOS ONE 2013;8:e57381.

16. Pan PD, Peter I, Lambert-Messerlian GM, Canick JA, Bianchi DW, Johnson $\mathrm{KL}$. Cell-free fetal DNA levels in pregnancies conceived by IVF. Hum Reprod 2005;20:3152-3156. 